\title{
An Ultra-flat Rectangular Optical Filter Based on Stimulated Brillouin Scattering in Fibre
}

\author{
Wei Wei ${ }^{(1)}$, Lilin $\mathrm{Yi}^{\left(1^{*}\right)}$, Yves Jaouën ${ }^{(2)}$, Yi Dong ${ }^{(1)}$,Weisheng $\mathrm{Hu}^{(1)}$ \\ (1) State Key Lab of Advanced Optical Communication Systems and Networks, Shanghai Jiao Tong \\ University, Shanghai 200240, China, *lilinyi@sjtu.edu.cn \\ ${ }^{(2)}$ Institut Telecom / Telecom ParisTech, CNRS UMR5141, 75634 Paris, France
}

\begin{abstract}
We demonstrate a rectangular optical filter based on stimulated Brillouin scattering with bandwidth from $50 \mathrm{MHz}$ to $4 \mathrm{GHz}$. The passband ripple is $\sim 1 \mathrm{~dB}$ by nonlinearity management of the pump lines and using fibre with a single Brillouin peak.
\end{abstract}

\section{Introduction}

With the rapid development of high speed optical transmission systems and microwave photonics, the requirements for flexible filtering and sub-band switching are in great need of rectangular optical filters with tunable bandwidth ranging from $\mathrm{MHz}$ to several $\mathrm{GHz}$. Utilizing the steep-edged optical filter, interference from adjacent bands can be suppressed at the extreme and the spectral efficiency will be further improved. Several methods have been proposed to obtain such kind of filters, including specially designed fibre Bragg gratings ${ }^{\prime}$, microring-based optical combs ${ }^{2}$, etc. However, there is still a great gap between the above filters and the ideal rectangular filter with an ultra-flat passband and a shape factor (SF) of 1.

Stimulated Brillouin scattering (SBS) in fibre has also been proposed to implement optical filters $^{3}$. In the previous work, we presented a narrowband rectangular SBS filter with very steep edge and tunable bandwidth from $50 \mathrm{MHz}$ to $3 \mathrm{GHz}$ based on digitally controllable electrical comb and a feedback compensation method $^{4}$. But with the increase of the filter bandwidth, the passband flatness deteriorated rapidly. The ripple was as high as $3.7 \mathrm{~dB}$ for 3$\mathrm{GHz}$ bandwidth, which was believed to be caused by the four-wave mixing (FWM) effect of the ultra-dense pump lines as well as the multiple SBS gain peaks of the used fibre. As a result, the available filter bandwidth was limited.

In this paper, we extend the maximum filter bandwidth to $4 \mathrm{GHz}$ with $15-\mathrm{MHz}$ tuning resolution. After using nonlinearity management to mitigate the FWM effect and utilizing a more suitable fibre with a single Brillouin gain peak, the in-band ripple is decreased to $\sim 1 \mathrm{~dB}$ for all bandwidth cases while the unwanted out-ofband gain is further suppressed. Based on this filter, we demonstrate a sub-band extraction of a multi-band orthogonal frequency division multiplexing (OFDM) signal.

\section{Principle}

Theoretically, in order to obtain the ideal rectangular gain spectrum, a pump consisting of equal-amplitude spectral lines with interval equaling the natural SBS gain bandwidth is required. In the previous work, we generated an electrical comb using an arbitrary waveform generator (AWG) and then modulated it on the light to generate the pump. Given the nonlinear responses of electrical and optical components, the flat electrical spectral lines led to uneven SBS gain as shown in Fig.1 (a), thus a feedback compensation was proposed to digitally control the amplitude of each electrical spectral line according to the measured SBS gain so as to optimize the shape of the targeted SBS filter.

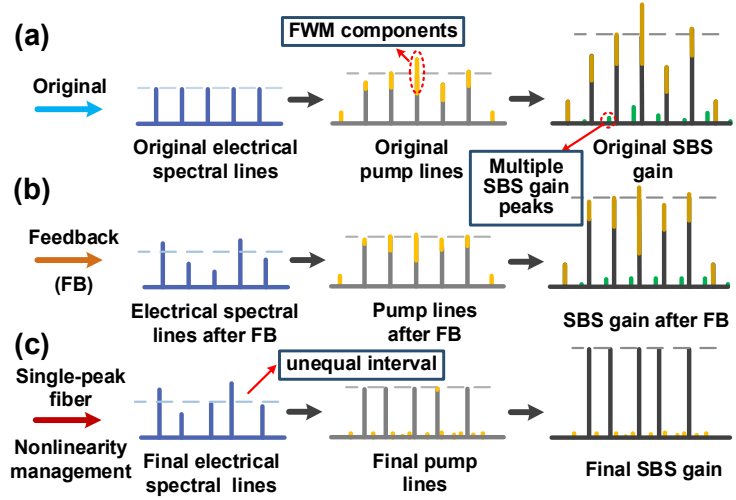

Fig. 1: The electrical spectral lines, pump lines and the SBS gain in different cases

In the feedback process it was assumed that the SBS gain at a certain frequency was only related to the corresponding electrical spectral line. It worked well when the filter bandwidth was small. However, with the increase of the filter bandwidth, the increasing pump power and number of spectral lines caused remarkable FWM which redistributed the pump power and generated new spectral lines at the stop band as shown in Fig. 1 (a) and (b) in the color of yellow. It resulted in a partial failure of the feedback and larger passband ripple. Therefore an effective 
nonlinearity management was required.

We propose a nonlinearity management method as shown in Fig. 1 (c). We set frequency interval of the electrical spectral lines randomly around the natural SBS gain bandwidth instead of the equal interval. In this case the new spectral lines generated by the FWM are no longer superposing on the original lines. As a result, the power of these new spectral lines are very small compared to the original lines and can just introduce tiny gain or even under the threshold of SBS effect. Therefore the flatness of the passband is greatly improved and the unwanted gain out of the passband can be partly suppressed as well.

Moreover, the natural SBS gain spectrum is related to the material doping profile. In the Germanium-doped G-652 fibre, there are 4 SBS peaks (i.e. four significant $L_{o m}$ acoustic modes) as sketched in Fig. 1 (a) and (b) in the color of green while the measured result is shown in Fig. 2 (a), which also contributes to the uneven filter passband. Therefore we employed a fibre with a single Brillouin peak as shown in Fig. 2 (b) which was highly desired in our experiment.
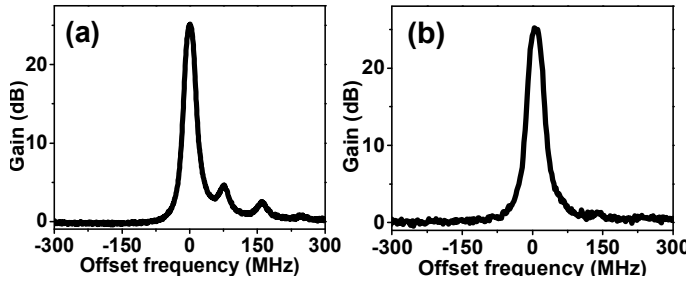

Fig. 2: The natural SBS gain in (a) G-652 fibre (b) fibre with a single Brillouin peak

\section{Experiment and results}

The experimental setup is shown in Fig. 3. An external cavity laser (ECL) operating at $1550 \mathrm{~nm}$ was split into two branches. In the upper branch, an AWG was used to generate the electrical spectral lines with random frequency interval within $\pm 1-\mathrm{MHz}$ deviation from the natural SBS bandwidth of $15 \mathrm{MHz}$, i.e. $14 \mathrm{MHz}, 15 \mathrm{MHz}$ and $16 \mathrm{MHz}$. Then it was modulated on the light to generate the SBS pump lines utilizing a dualparallel Mach-Zehnder modulator (DPMZM). With proper phase control of the driving signals and bias control of the DPMZM, the optical carrier-suppressed single sideband (O-CS-SSB) modulation was achieved. The O-CS-SSB signal was then amplified by an erbium doped fibre amplifier (EDFA) and launched into the $12.5 \mathrm{~km}$ single-peak fibre through an optical circulator. In the lower branch, a sweeping signal covering the whole SBS gain region from an electrical vector network analyzer (EVNA) was modulated on the light to generate the probe signal. An optical bandpass filter (BPF) removed the left sideband of the probe signal for stable SBS gain measurement. Then the probe light went through the single-peak fibre and was amplified once it swept within the SBS gain region. A polarization controller (PC3) was used to achieve the maximum SBS gain. After the SBS process, the probe signal was detected by a photodiode (PD) and then sent into the EVNA. The amplitude and phase response were measured by the EVNA and the SBS gain spectrum could be obtained by subtracting the results with and without the SBS amplification.

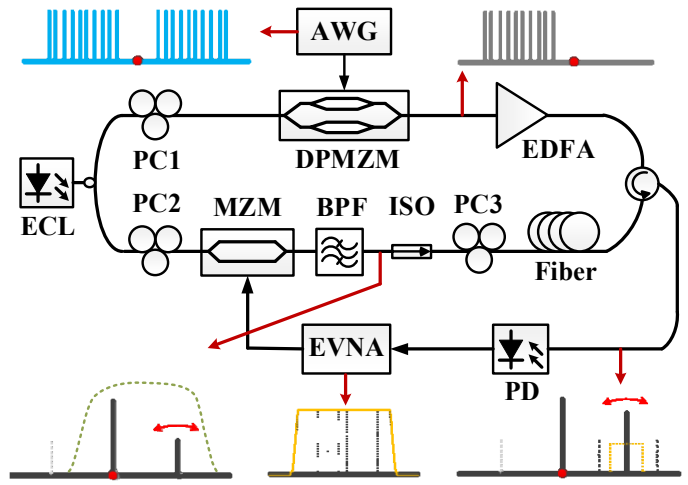

Fig. 3: Experimental setup
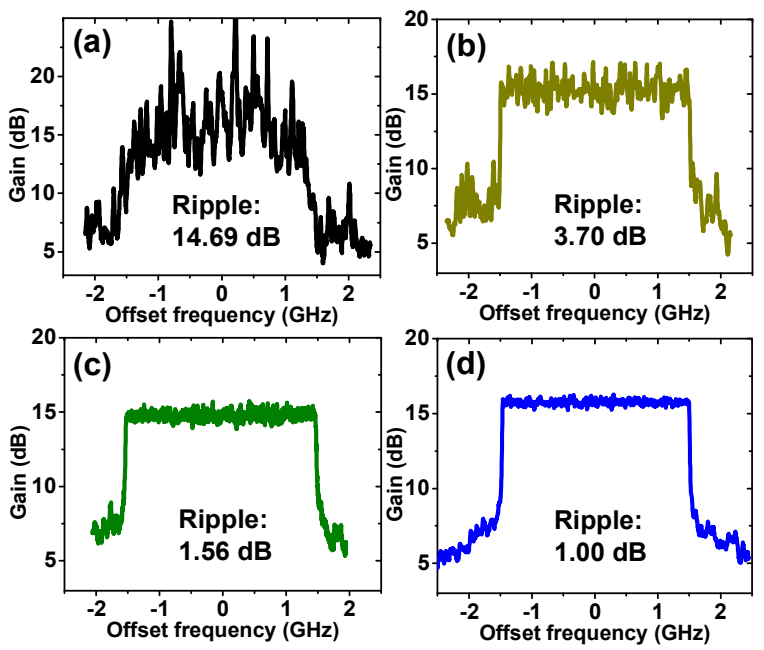

Fig. 4: The measured SBS spectra (a) original spectrum (b) after feedback process (c) utilizing nonlinearity management (d) utilizing nonlinearity management with single-peak fibre

The measured SBS spectra with $3-\mathrm{GHz}$ bandwidth in different cases are shown in Fig. 4. The original SBS gain generated by the electrical spectral lines with equal amplitude and equal frequency interval were seriously affected by the FWM and nonlinear response of the electrical and optical components. As shown in Fig. 4 (a), the SBS gain was far from the rectangular shape. When implementing the feedback compensation as shown in (b), the ripple was still as high as $3.70 \mathrm{~dB}$ with large unwanted gain out of the passband. After utilizing the unequal interval spectral lines, the passband ripple in (c) was significantly reduced to $1.56 \mathrm{~dB}$. By using the single-peak fibre, the 
ripple was further decreased to $1.00 \mathrm{~dB}$ shown in (d) and the gain at stop band was smaller than the previous cases. We can further decrease the passband ripple by implementing more feedback iterations, but $\sim 1 \mathrm{~dB}$ ripple already satisfies most of the applications.

The bandwidth of the proposed filter can be changed flexibly with a resolution of $15 \mathrm{MHz}$ by changing the number of the electrical spectral lines generated by the AWG. The gain spectra and phase responses of different filter bandwidths ranging from $50 \mathrm{MHz}$ to $4 \mathrm{GHz}$ are shown in Fig. 5 (a) and (b) respectively. In (c) and (d) the passband ripple and filter gain are compared among 3 conditions: only using feedback compensation (FB), using both FB and nonlinearity management (NM), using $F B$ and NM in the single-peak fibre (SPF). The figures illustrate that in the third case the SBS filter has the largest gain and smallest ripple thus proving the validity of the NM in the SPF.
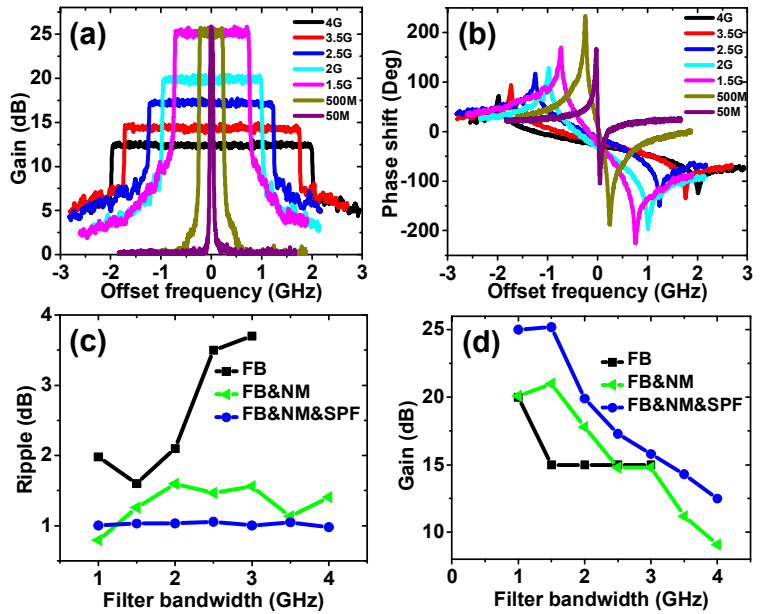

Fig. 5: SBS filters with different bandwidth (a) amplitude response (b) phase response (c) passband ripple and (d) filter gain

For the SBS filter shown in Fig. 6 (a) and (b) with 1-GHz bandwidth, the passband ripple was only $1.00 \mathrm{~dB}$ thus leading to a very smooth phase response. Meanwhile the shape factor $\left(\mathrm{SF}_{15 \mathrm{~dB}}\right)$ was 1.075 which was almost the ideal rectangular case. Based on this filter, we demonstrated a typical application of OFDM sub-band extraction as shown in Fig. 6 (c). We first generated a double-sideband OFDM signal containing 3 sub-bands. The bandwidth of each sub-band was $1 \mathrm{GHz}$ and the band gap was as narrow as $100 \mathrm{MHz}$. Then we used the proposed filter to amplify only the middle subband by $25 \mathrm{~dB}$. And just small parts of the adjacent bands were amplified slightly thus making a $\sim 21-d B$ distinction between the middle sub-band and the adjacent bands. The extraction can be quite flexible thanks to the filter flexibility of the bandwidth and wavelength.
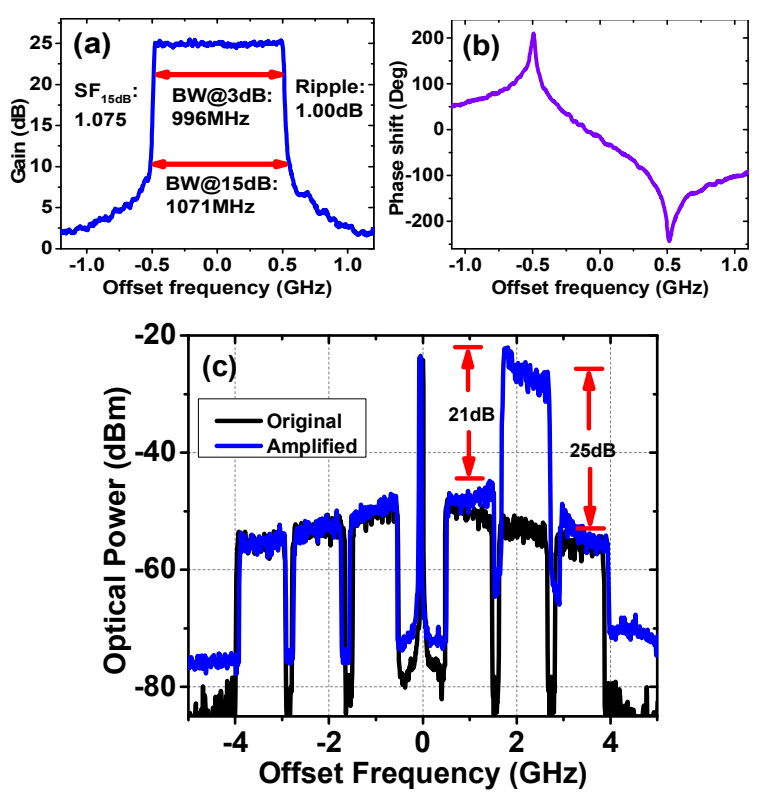

Fig. 6: The (a) amplitude and (b) phase response of 1-GHz filter, (c) SBS filter application of sub-band extraction

\section{Conclusions}

We have presented an ultra-flat rectangular optical filter based on stimulated Brillouin scattering and demonstrated a typical filter application of OFDM sub-band extraction. We propose the method of utilizing unequal interval spectral lines to mitigate the FWM in the fibre with only a single Brillouin gain peak. As a result, the passband ripple is decreased to as low as $\sim 1 \mathrm{~dB}$. With flexible bandwidth from $50 \mathrm{MHz}$ to 4 $\mathrm{GHz}$ and high tuning resolution of $15 \mathrm{MHz}$, the proposed steep-edged rectangular filter can find versatile applications in optical and microwave signal processing.

\section{Acknowledgements}

This work was supported by Nature Science Foundation China (611322507, 61132004) and Program of Excellent PhD in China (201155).

\section{References}

[1] X. Zou et al., "All-fiber optical filter with an ultranarrow and rectangular spectral response," Opt. Lett., Vol 38, no. 16, p. 3096 (2013).

[2] X. Xue et al., "First demonstration of a tunable singlebandpass photonic radiofrequency filter based on optical frequency comb from a microring," Proc. OFC, Tu2A.7, San Francisco (2014).

[3] T. Sakamoto et al., "Low distortion slow light in flat Brillouin gain spectrum by using optical frequency comb," Opt. Express, Vol 16, no. 11, p. 8026 (2008).

[4] W. Wei et al., "A bandwidth-tunable narrowband rectangular optical filter based on stimulated Brillouin scattering," Proc. OFC, W4F.5, San Francisco (2014).

[5] Y. Jaouën et al., "Stimulated Brillouin scattering in specialty optical fibers: importance of material, structure and manufacturing parameters," Proc. CLEO, CF3N.1, San Jose (2012) 